Sharif University of Technology
Scientia Iranica
SCIENTIA

Research Note

\title{
A robust proportion-preserving composite objective function for scale-invariant multi-objective optimization
}

\author{
M. Daneshmand ${ }^{a}$, M. Tale Masouleh ${ }^{\mathrm{b}, *}$, M.H. Saadatzi ${ }^{\mathrm{c}}$, C. Ozcinar $^{\mathrm{d}}$ and \\ Gh. Anbarjafari ${ }^{a}$ \\ a. iCV Group, Institute of Technology, University of Tartu, Tartu 50411, Estonia. \\ b. Laboratory of Human and Robot Interaction, Faculty of New Sciences and Technologies, University of Tehran, Tehran, Iran. \\ c. Department of Mechanical Engineering, Colorado School of Mines, USA. \\ c. Telecom ParisTech, Paris, France.
}

Received 8 June 2016; accepted 21 November 2016

\author{
KEYWORDS \\ Proportion-Preserving \\ Composite Objective \\ Function (PPCOF); \\ Multi-objective \\ optimization; \\ Pareto-optimal set of \\ solutions; \\ Non-dominated \\ Sorting Genetic \\ Algorithm II (NSGI- \\ II); \\ Planar Parallel \\ Mechanisms (PPMs).
}

\begin{abstract}
This paper aims to introduce a proportion-preserving composite objective function for multi-objective optimization, namely, PPCOF, and validate its efficiency through demonstrating its applicability to optimization of the kinetostatic performance of planar parallel mechanisms. It exempts the user from both specifying preference factors and conducting decision-making. It consists of two terms. The first one adds the normalized objective functions up, where the extrema result from single-objective optimization. To make the composite objective function steer the variations of the objective functions while preserving rational proportions between them, as the main contribution of the paper, it is sought that the normalized objective functions take closely similar values, to which end they are juxtaposed inside a vector, which is then scaled such that its Euclidean norm-2 is equal to that of the vector of all ones with the same dimensions. Then, the second term is constructed as the addition of penalty factors standing for the absolute value of the difference between each element of the foregoing vector from 1. From the obtained results, with considerably smaller computational cost, the PPCOF obtains an optimal solution that is not dominated by any point from a set of Pareto-optimal solutions offered by NSGA-II. (C) 2017 Sharif University of Technology. All rights reserved.
\end{abstract}

\section{Introduction}

Numerous industrial applications and analytical simulative studies require dealing with nonlinear programming intended to solve Multi-Objective Optimization Problems (MOOP) due to the significant roles they play in making decisions leading to fair settlements

\footnotetext{
*. Corresponding author. Tel.: +982161118413; Fax: +982188497324

E-mail addresses: $\{$ mortezad, shbg\}@ut.ee ( $M$.

Daneshmand); m.t.masouleh@ut.ac.ir (M. Tale Masouleh); msaadatz@mymail.mines.edu (M.H. Saadatzi); cagri.ozcinar@telecom-paristech.fr (C. Ozcinar)
}

doi: $10.24200 /$ sci. 2017.4313 between conflicting criteria taken into account in the design, planning, and control stages [1-5]. More clearly, practical applications are usually aimed at achieving more than one goal simultaneously, where satisfying each objective to the greatest extent demands ruining the performance or desirability in terms of others. Overcoming the foregoing conflict and accomplishing an optimized solution that would result in a reasonable compromise among the aforementioned contradictory objectives is, in fact, the main goal, hence the underlying challenge in the context of MultiObjective Optimization (MOO) [6-8]. One of the fundamental questions as regards this issue is how, and on what basis, to decide to which degree each objective function's enhancement upon adjusting the 
optimization variables should be allowed to degrade the others. In simpler terms, due to the fact that MOO processes are mostly devised such that they could handle real-life applications, which vary significantly in terms of both the proportions between the importance and significance ratings of the criteria and the scales and units they are represented through, as long as they are treated in the same manner, i.e. regardless of the settings associated with the foregoing notions, the inferences might be considerably, and practically unacceptably, inauthentic. First, they may be sensitive to the representations of the parameters, meaning that the conclusion made is applicable only to the particular case-study taken into account, but most likely, not to others. Another drawback of the latter strategy is that it ignores the significance level of each objective, which is, however, of paramount importance when it is put into practice [9-11].

Many preference-based MOO algorithms are based on combining all the criteria at hand through scalarizing them and coming up with a single criterion, followed by performing Single-Objective Optimization (SOO) as a special case of MOO, since it has basically been deemed both mathematically and computationally more manageable than MOO [12-14]. In other words, for the sake of avoiding the aforementioned deficiencies and misinterpretations, the assumption has been that the degree to which the significance of each objective function should affect the way the optimization procedure is steered could be represented by a weight, i.e. preference factor, having been determined while also noticing the scale and unit utilized for measuring and reporting the associated quantity.

On the other hand, Evolutionary Multi-objective Optimization (EMO), as an Evolutionary Algorithm (EA), is intended to deal with all the objectives at once, which should stand higher chances of satisfying all the objectives fairly and reliably [15-20]. The above strategy results in a set of Pareto-optimal solutions, being supposedly maximally wide in terms of every objective, each pair among which offers a trade-off between the conflicting criteria [11-21]. Accomplishing maximally-spread multiple solutions was not guaranteed by traditional approaches, because of the demand for more than once implementing the same algorithm with different settings [22-25].

Although EMO processes mostly result in a set of solutions, even if it is distributed in the best manner possible, practically, a single solution has to be determined and utilized. In other words, each point from the set of solutions stands for a specific order of the significances of the objectives taken into account, where it is left to the user's taste or discretion to decide which one would best satisfy the requirements in a real-world context, perhaps differing considerably from case to case. This gives rise to a demand for decision-making algorithms [26] to succeed them [2729], which are prone to subjectivity, and have to be conducted in a qualitative, rather than quantitative, manner, in order to opt for the solution that would best suit the particular conditions associated with the application [30-33].

Although EMO algorithms bring about the advantage of leaving various solution options at one's disposal, in fact, it can be asserted that they suffer from the lack of immediate practical importance, since there is no solid, standard procedure through which the final decision could be made. To be more clear, the fact that they demand performing decision-making processes on the basis of higher-level information, which is particular to the use-case, makes them fundamentally insufficient, in that the user is exposed to uncertainty regarding the optimality of the choice. Not only is the foregoing higher-level information usually debatable and mathematically unmeasurable, but also a common, more awkward challenge is that, often times, a user hardly possesses enough knowledge, experience, or expertise to provide it.

There are numerous EMO methods proposed and implemented in the literature, such as the Paretosolutions-based approach known as Non-dominated Sorting Genetic Algorithm-II (NSGA-II) in [20], all of which entail the aforementioned deficiency, namely, the fact that they necessitate the use of decisionmakers to decide on the best possible solution. On the other hand, as stated before, preference-based algorithms devised for such a purpose suffer from subjectivity and qualitativeness. This paper is intended to circumvent the shortcomings of evolutionary and preference-based viewpoints simultaneously through introducing a Proportion-Preserving Composite Objective Function (PPCOF) which suggests a robust, realistic criterion for the search process, which can be performed using an arbitrary SOO approach. On top of the fact that the decision-making process is not required anymore by the $\mathrm{PPCOF}$, it brings about the advantage that upon obtaining the knowledge of the rational proportions of the objective functions to each other, a logical trade-off between them is ensured by maintaining the same proportions, since the PPCOF guides the optimization procedure such that all of them are satisfied to a closely similar extent and located in normally desirable intervals within the decision variables space.

The aforementioned knowledge, i.e. information on the desired proportions of the objective functions to each other, is obtained through SOO processes considering each individual separately, rather than using the nominal extrema. The latter guarantees that the proportions are determined neither subjectively nor qualitatively, which are enforced by the PPCOF via normalizing each objective function based on the 
aforesaid extrema, i.e. the ones found through SOO procedures as well as introducing penalty factors demanding that the normalized objective functions take quite similar vales in the end. By PPCOF, this requirement is given the same priority as the main concept of optimization, i.e. the desire for the objective functions themselves getting as close to their optimal values as possible.

The PPCOF makes the optimization algorithm search through all the possibilities and suggests a definitive best solution. Through normalizing each objective function based on its practical extrema and incorporating penalty factors standing for the differences between them, it is guaranteed that they are all deemed to have the same level of importance, and the best solution returned is in line with the ideal vector, which is constructed by juxtaposing the ideal values each of which would take in the absence of the others. Noticing that, as aforementioned, in most practical contexts, different objective functions are reported in non-homogeneous units or with different scales, using the foregoing strategy, the effect of these complications is overcome, and the composite objective function is made robust against the changes of the scales by which the objective functions are represented, and each of them plays a role as significant as the others in guiding the algorithm through the search process. Besides, the practical reliability of the PPCOF is demonstrated via applying it to a use-case, namely, the MOO of the point-displacement and rotational kinematic sensitivity [34,35] and workspace of eight Planar Parallel Mechanisms (PPMs), and comparing its efficiency with that of NSGA-II, which has been implemented on the same case-study in [36].

The remainder of this paper is organized as follows. First, the notion of MOO is described, being followed by a review of the existing methods aimed at solving MOOPs. Then, the PPCOF is introduced, and mathematically expressed. Afterward, the specifications of the case-study utilized for validating the PPCOF are discussed. Next, the case-study is implemented using Differential Evolution (DE) [37], and the results are presented along with discussion as to how they would demonstrate the efficiency of the PPCOF. Finally, the paper is concluded through summarizing the content.

\section{Overview of $\mathrm{MOO}$}

In this section, the basic structure of common MOO algorithms is reviewed, which requires defining a collection of basic conceptual and mathematical terminologies, being presented as follows.

As per the normal practice, which is also understandable from the mathematical nature of the problem, a multi-objective optimization procedure should be based on a multi-dimensional space, which is called the objective space [11]. The foregoing space often does not make geometric sense.

Following a notation similar to that of [11], upon denoting the above multi-dimensional space as $\mathcal{Z}$, for each $n$-dimensional solution vector $\mathbf{x}$ to the MOOP, a point corresponding to it can be found in the $m$ dimensional objective space, such that:

$$
f(\mathbf{x})=\mathbf{z}=\left[\begin{array}{llll}
z_{1} & z_{2} & \ldots & z_{m}
\end{array}\right]^{\mathrm{T}}
$$

where the search takes place in the $n$-dimensional decision variables space, and the preference factors are juxtaposed inside preference vector $\mathbf{w}$, such that:

$$
\mathbf{w}=\left[\begin{array}{llll}
w_{1} & w_{2} & \cdots & w_{m}
\end{array}\right]^{\mathrm{T}},
$$

where $w_{i}, i=1,2, \ldots, m$, ith represents the preference factor associated with the $i$ th objective function.

By means of finding optimal solution $\mathbf{x}_{i}^{*}, i=$ $1,2, \ldots, m$, to the SOO procedure optimizing the $i$ th objective function, namely, $f_{i}$, one could define the ideal vector $\mathbf{z}^{*}$, such that:

$$
\mathbf{z}^{*}=\mathbf{f}^{*}=\left[\begin{array}{llll}
f_{1}^{*} & f_{2}^{*} & \cdots & f_{m}^{*}
\end{array}\right]^{\mathrm{T}},
$$

where $f_{i}^{*}=f_{i}\left(\mathbf{x}_{\mathbf{i}}^{*}\right)$. More clearly, $\mathbf{x}_{\mathbf{i}}^{*}$ is the solution to the following Single-Objective Optimization Problem (SOOP):

$$
\begin{array}{ll}
\text { Minimize } & f_{i}(\mathbf{x}), \\
\text { subject to } & \mathbf{x} \in \mathcal{S} .
\end{array}
$$

in which $\mathcal{S}$ denotes the decision variables space.

Obviously, the ideal vector does not exist in the objective space, i.e. $\mathbf{z}^{*} \notin \mathcal{S}$, since otherwise, there would not be any conflict between the objectives, i.e. they could all be satisfied to the greatest extent at the same time, and the solution to all the SOOPs would be the same, in which case the fundamental notion of MOOPs would be violated. Nevertheless, an ideal solution to the MOOP would be the one as close as possible to the ideal vector, which is the underlying hypothesis of the PPCOF as well, being described thoroughly, both mathematically and conceptually, in the upcoming sections.

Other sorts of objective vectors may also be defined for different purposes. For example, the Nadir objective vector [38], $\mathbf{z}^{\text {nad }}$, which consists of the highest values the objectives could take inside the Paretooptimal space (not to be confused with the whole objective space), is aimed at normalizing the objective space within a subspace defined on the basis of the ideal vector, i.e. the minimal values the objectives could take, as the lower bound and the worst values, on the other hand, as the upper bound. The latter, using the preceding notation consistently, could be mathematically expressed as follows: 


$$
f_{i}^{\text {nad }}=\frac{f_{i}-f_{i}^{*}}{z_{i}^{\text {nad }}-f_{i}^{*}}, \quad i=1,2, \ldots, m
$$

where $f_{i}^{\text {nad }}$ is the $i$ th Nadir objective function. Moreover, in the above relation, $z_{i}^{\text {nad }}$ is the highest value that the $i$ th objective function could take. In other words, $\left[\mathbf{z}^{\text {nad }}\right]_{(i)}=z_{i}^{\text {nad }}$.

It should be noted that most MOO methods are based on determining the dominance of each solution vector over the others while searching for the optimal one through a definite population $[39,40]$. The concept of dominance, in the context of this paper, is defined such that each element of the dominant vector is not worse than the corresponding element in the other one, where at least one element from the dominant vector must be preferable to its counterpart.

In mathematical terms, if $\mathbf{x}_{1}$ and $\mathbf{x}_{2}$ are members of the decision variables space, then the dominance of $\mathbf{x}_{1}$ over $\mathbf{x}_{2}$, i.e. $\mathbf{x}_{1} \triangleleft \mathbf{x}_{2}$, means that the following conditions must hold:

$$
\forall i, i \in\{1,2, \ldots, m\} \Longrightarrow f_{i}\left(\mathbf{x}_{\mathbf{1}}\right) \preceq f_{i}\left(\mathbf{x}_{\mathbf{2}}\right),
$$

and:

$$
\exists i\left(i \in\{1,2, \ldots, m\} \wedge f_{i}\left(\mathbf{x}_{\mathbf{1}}\right) \prec f_{i}\left(\mathbf{x}_{\mathbf{2}}\right)\right),
$$

where signs $\preceq$ and $\prec$ stand for element-wise comparison. It should be noted that the above relations do not necessarily hold between every pair of solutions, i.e. the pair may be non-dominant to each other.

Thus, noticing that dominating other solutions means being "better" from optimization point of view, the final goal of any MOOP is to try to find the non-dominated solution. More clearly, the best solution is the one that is not dominated by, i.e. inferior to, any other solution.

Based on the above definition of dominance, the set of Pareto-optimal solutions to an MOOP with a finite decision variables space should be the minimal collection within which one is no longer able to derive any preference. Besides, it has to contain all the solutions necessary for dominating the rest of the decision variables space. In formal words, the Paretooptimal solutions set:

- Must consist of solutions, each pair of which does not satisfy any dominance relation;

- Contain at least one solution that dominates it for every solution outside it.

Mathematically, the set of Pareto-optimal solutions, $\mathcal{P}=\left\{\mathrm{x}_{1}, \mathrm{x}_{2} \ldots, \mathrm{x}_{\mathrm{p}}\right\}$, is defined based on two constraints as follows:

$$
\begin{aligned}
& \forall i \forall j, i \in\{1,2, \ldots, p\} \wedge j \in\{1,2, \ldots, p\} \wedge i \neq j \\
& \Longrightarrow \mathbf{x}_{\mathbf{i}} \not \mathbf{x}_{\mathbf{j}} \wedge \mathbf{x}_{\mathbf{j}} \not \mathbf{x}_{\mathbf{i}},
\end{aligned}
$$

and:

$$
\forall \mathrm{x}_{\mathrm{i}}, \mathrm{x}_{\mathrm{i}} \in \mathcal{S} \wedge \mathrm{x}_{\mathrm{i}} \notin \mathcal{P} \Longrightarrow \exists \mathrm{x}_{\mathrm{j}}\left(\mathrm{x}_{\mathrm{j}} \in \mathcal{P} \wedge \mathrm{x}_{\mathrm{j}} \triangleleft \mathrm{x}_{\mathrm{i}}\right) .
$$

The manner through which the set of non-dominated solutions is found should be chosen based on the specifications of the MOOP under study, for which various strategies have been suggested in the literature.

As an instance, finding the best non-dominated front has been investigated in [41], which determines the target non-dominated set of solutions by means of comparing the elements in the existing set with all the others and updating the set iteratively, upon replacing the elements by the ones that dominate them, but are not included in the set heretofore, along with assimilating the outsider elements that are not dominated by any element already included in the set.

Sorting algorithms for finding non-dominated sets of solutions [42], on the other hand, follows the above procedure by excluding the member elements of the best non-dominated solution set and applying the same process to the rest, resulting in a second non-dominated front. Next, the latter front is excluded, and the same thing is implemented on the remaining solutions, in order to find the third non-dominated front. This process is iterated until the worst non-dominated front is found, and every solution is categorized into one of the designated classes, i.e. assigned to a non-dominated front.

\section{Review of the existing literature on MOO methods}

In this section, the methods proposed for the purpose of solving MOOPs are broadly categorized and explained. As aforementioned, MOO procedures, from a wide perspective, could be classified into two sorts, namely, preference-based and evolutionary ones. In what follows, examples of the latter types of MOO algorithms will be reviewed and briefly discussed.

EMO techniques make use of search algorithms looking for non-dominated solutions, reviewed in the previous section. NSGA-II is a well-known example of the foregoing type, which employs an elitist principle, along with a diversity-preserving mechanism. With the aid of basic Genetic Algorithm (GA) [14] operators, it produces the offspring population based on the parent population, then merged and classified through nondominated sorting. The non-dominated fronts are next used to fill a new population, where by the time that there is not enough room to accommodate all the members of the current front, the ones kept in are those preserving the diversity as much as possible, according to a metric called croding distance [43]. Afterward, a crowding tournament selection operator is applied, which checks two conditions, namely a 
non-domination rank and a normalized search space around each solution. The former is responsible for determining a preference between the given arguments based on the non-dominated front they belong to, and the latter prevents solutions from the same front, in which case the one possessing a larger crowding distance will win the tournament.

Among instances of classical approaches, on the other hand, the most commonly used ones are preference-based methods which multiply each objective function by a preference factor and create a single composite objective function to undergo SOO. More clearly, the weight multiplied by each objective function is the result of the multiplication of the coefficient used for adjusting the scale of the unit through which the objective is represented by an importance factor, both of which depend on the context the MOOP is associated with.

Determining the scalarizing factor is less tricky, since for the sake of ensuring that every objective is homogeneously presented and its influence does not bear unit-related information, it can be normalized within the range specified by the extremal values it can take. Mathematically, the resulting SOOP, which is, in fact, based on objective function, $f$, standing for a weighted sum of the normalized objectives, can be expressed as follows:

$$
\text { Minimize } f(\mathbf{x})=\sum_{i=1}^{m} w_{i} f_{i}^{\text {norm }}(\mathbf{x}),
$$

subject to $\mathrm{x} \in \mathcal{S}$,

where, as could be found in Eq. (2), $w_{i}$ is the preference factor assigned to the $i$ th objective function, and $f_{i}^{\text {norm }}$ denotes its normalized form, i.e.:

$$
f_{i}^{\text {norm }}(\mathbf{x})=\frac{f_{i}(\mathbf{x})-f_{i}^{\min }(\mathbf{x})}{f_{i}^{\max }(\mathbf{x})-f_{i}^{\min }(\mathbf{x})}, \quad i=1,2, \ldots, m,
$$

where $f_{i}^{\min }$ and $f_{i}^{\max }$ are, respectively, the minimal and maximal values of $f_{i}(\mathbf{x})$ for $\mathbf{x} \in \mathcal{S}$.

As aforesaid, the most challenging issue with the weighted-sum method is the question regarding how the weights have to be determined, which renders the solution unreliable. The relation between the solution to the SOOP of Eq. (10) and the Pareto-optimal set has been investigated in a number of studies, including [4446], where it has been shown that the former is a member of the non-convex portion of the Pareto-optimal front, which does not include an optimal solution to the optimization problem of Eq. (10).

In order to resolve the above problem, the $\varepsilon$ constraint approach could be employed. Herein, the values which objective functions take in the varying ranges are manipulated, and they confound us based on a specific set of criteria. More clearly, the revised MOOP would be described as follows:

$$
\text { Minimize } f(\mathbf{x})=\sum_{i=1}^{m} w_{i} f_{i}^{\text {norm }}(\mathbf{x})
$$$$
\text { subject to } \mathbf{x} \in \mathcal{S}, f_{i}^{\text {norm }}(\mathbf{x})<\varepsilon_{i} \text {, }
$$$$
i=1, \ldots, m \text {, }
$$

where $0 \leq \varepsilon_{i} \leq 1$ stands for the highest value the $i$ th objective function is allowed to take. For example, the method suggested in [47] takes one of the objectives into account and considers other constraints for the resulting MOOP.

Although the main drawback of generic weightedsum methods is alleviated by introducing the above constraints, the $\varepsilon$-constraint method is widely accused of entailing two main drawbacks. First, the solution is largely dependent on the constraints, which are demanded to be specified by the user, meaning that higher number of objectives will necessitate more input from the user. Besides, determining them is not a routine task, for which, no solid criterion or process has been suggested. In practice, if not enough attention is paid to the manner through which the values of the constraints are specified, the resulting options for the optimal solution would be either too various or too limited. More clearly, upon taking the strictest constraints into account, the MOOP might end up leading to no solutions at all, and, on the other hand, assigning the constraints too generously may result in all the members of the objective space being solutions to the MOOP, i.e. rendering the constraints completely powerless in terms of guiding the MOO procedure.

\section{The PPCOF}

As aforementioned, preference-based approaches have the virtue of coming up with a single solution deemed the best, where their main deficiency is that they depend on the discretion of the user and require, perhaps, considerable amount of input from the user to consider that, a reliable criterion is not necessarily always available. On the other hand, evolutionary algorithms, although initially seem to be automatic in the sense that they do not demand assigning preference factors, are not of direct practical use, since, after implementing them, a separate decision-making process is needed to determine the best choice amongst a set of Pareto-optimal solutions, which, again, incurs the necessity of manual contribution from the user.

The PPCOF tries to find a compromise between preference-based and Pareto-solution-based evolutionary MOO methods, which takes advantages of both, while overcoming the drawbacks entailed simultaneously. More clearly, it deals with the MOOP, such that a single optimal solution is found without requiring any input from the user, which means that both the 
demand for decision-making and the subjectivity in approaching the problem are removed. The underlying notion leading to the foregoing accomplishment lies in the idea that the values the normalized objective functions take are compelled to be similar to each other, i.e. the proportions between the values of the individual objective functions are checked to be similar to those of the ideal vector in order to avoid solutions that actually do minimize the value associated with the composite objective function, but are, orientation-wise, too far from what is expected in reality.

Although the search frameworks to be utilized along with the PPCOF are tantamount to those of preference-based methods, the need to provide the preference factors by the user is obviated through introducing penalty factors, necessitating that all the objective functions have to be satisfied to roughly the same extent. Mathematically, if the normalized objective functions had taken the same values, which is desired, the vector constructed by juxtaposing them would have had the same direction as that of the vector of all ones with the same dimensions, which would have a Euclidean norm- 2 of $\sqrt{m}$. Therefore, in the context of the PPCOF, each of the aforementioned penalty factors stands for the absolute value of the distance between the corresponding objective function and 1 , where it is scaled such that the Euclidean norm- 2 of the resulting vector of normalized objective functions is equal to $\sqrt{m}$. In order to ensure that the latter criterion is paid due attention, by the PPCOF, the penalty factor associated with each normalized objective function is treated in the same manner as itself, i.e. just added to it, meaning that the wish for the objective function taking a value as close to the desired one as possible is considered equally important to the demand for it being similar to the others.

Besides, the basic information for normalization is obtained by SOO procedures preceding the MOO, finding the associated extrema, rather than taking the nominal ones into account, which is necessary for preventing the unrealistic marginal values from causing misinterpretations. The latter strategy would guarantee that the composite objective function is robust against the scales and types of the objective functions at hand. All in all, the PPCOF consists of two terms, the first of which adds the normalized objective functions up as they are, and the second one is the summation of the aforementioned penalty factors, being, overall, mathematically expressed as follows:

$$
\begin{aligned}
\operatorname{PPCOF}(\mathbf{x})= & \sum_{i=1}^{m}\left(f_{i}^{\text {norm }}(\mathbf{x})\right. \\
& \left.+\left|\frac{\sqrt{m}}{\left\|\mathbf{f}^{\text {norm }}(\mathbf{x})\right\|_{2}} f_{i}^{\text {norm }}(\mathbf{x})-1\right|\right),
\end{aligned}
$$

which could be dealt with using an arbitrary SOOP approach. In the above relation, $\|$.$\| returns the$ Euclidean norm of its vector argument, and $\mathbf{f}^{\text {norm }}(\mathbf{x})$ denotes the vector of normalized objective functions as follows:

$$
f^{\text {norm }}(\mathbf{x})=\left[\begin{array}{llll}
f_{1}^{\text {norm }}(\mathbf{x}) & f_{2}^{\text {norm }}(\mathbf{x}) \ldots f_{m}^{\text {norm }}(\mathbf{x})
\end{array}\right]^{\mathrm{T}}
$$

It is worth noticing that if any of the normalized objective functions, $f_{i}^{\text {norm }}(\mathbf{x}), i=1,2, \ldots, m$, is supposed to get maximized, despite the default assumption of getting minimized, $\hat{f}_{i}^{\text {norm }}(\mathbf{x})=1-f_{i}^{\text {norm }}(\mathbf{x})$ shall replace it in Eqs. (13) and (14), which preserves the interval specified by the associated minimum and maximum values, but at the same time, implicitly conveys the foregoing notion by reversing the trend of the changes of the corresponding objective function based on the variations of the decision variables.

\section{Review of the case-study: MOO of the point-displacement and rotational kinematic sensitivity and workspace of PPMs}

In this section, MOO of the point-displacement and rotational kinematic sensitivity and workspace of PPMS is considered as a case study for the sake of verifying the reliability of the PPCOF. It is worth noticing that the underlying motivation for choosing this case study, i.e. what qualifies it as an MOOP, is the fact that it comprises objectives being in conflict with each other [48]. More clearly, optimizing the design parameters using SOO procedures aiming at maximizing the area or volume of the workspace [49,50], in most cases, will result in noticeably weak kinetostatic performance, which is represented, in the context of this paper, by kinematic sensitivity, and functionally disturbing levels of discontinuity and singularity, where those leading to great kinematic sensitivity usually suffer from extremely limited workspaces, which are not practically useful. More clearly, in the latter case, the optimization process naturally tends to limit the workspace to the point or region that denotes the best possible kinematic sensitivity.

The goal of this case study is, in fact, to try the PPCOF out and check whether the proposed composite objective function would actually both compensate for the absence of preference factors that would otherwise be expected from the user in preference-based MOO settings and deal with all the objectives fairly and logically without demanding performing decision-making. More clearly, if the solution found upon utilizing the PPCOF is not dominated by any of the ones obtained by implementing Pareto-solutions-based MOO on the same case study, and is preferable, or at least close, to them, then it means that the PPCOF has been devised, such that it could accomplish its missions that were 
sought from the outset. The relations and concepts required for dealing with the case study are explained in detail in studies such as [51]. Nevertheless, for quick reference, they are briefly recalled in what follows.

Various efficiency indices have been proposed for the evaluation and comparison of Parallel Mechanisms (PMs) [52-54]. However, most of them entail several drawbacks and, as a result, are neither universally reliable nor physically applicable. Numerous studies reported in the literature have asserted and demonstrated that most of the proposed kinetostatic performance indices do not indicate the accuracy of PMs in a physically sound manner $[55,56]$. For instance, some of them consider input and design errors simultaneously and are not able to distinguish between them $[57,58]$.

Since the translational and rotational parts of Jacobian matrices are not unit-consistent, the performance indices defined on the basis of this matrix, as well as the characteristic length proposed in [59], are not physically applicable, since changing the scale of geometric properties could change their results significantly. This assertion is investigated extensively and proved in $[36,55,56,60,61]$. Therefore, two separate performance indices are considered for the purpose of this paper, namely point-displacement and rotational kinematic sensitivity, which have been recently proposed to the end of alleviating the limitations of the above notorious indices [55]. However, according to the classification proposed in $[62,63]$, this index is posture-dependent. Therefore, its posture-independent counterpart, the global kinematic sensitivity [64], is considered, which measures the performance of the mechanical system in the whole workspace and gives a meaningful and convincing value.

According to the study carried out in [65], there are ten different feasible types of PPMs. However, from the study conducted in [36], it is revealed that even an optimized 3-RPP or 3-RPP has a limited workspace. Therefore, they are excluded from the rest of the paper, and the remaining eight are considered. The latter PPMs are schematically shown in Figure 1, along with their corresponding geometrical design parameters, where each architecture comprises three kinematically identical limbs. Throughout this paper, prismatic and revolute joints are denoted by $\mathrm{P}$ and $\mathrm{R}$, respectively. With this notation, the kinematic arrangement of the joints in each leg is shown by juxtaposing the joint symbols in the same order as they appear from the base to the end-effector, where the actuated joint is underlined.

It is worth mentioning that PMs usually comprise various working modes. However, trajectory planning while switching between different working modes, in most cases, is a demanding task, which regularly necessitates passing through singular regions. Therefore, in this paper, only the working mode that possesses the best kinetostatic performance is taken into account for each PPM when calculating its input-output velocity relation [65], which can be expressed as follows:

$$
\left[\begin{array}{c}
\zeta_{1}^{\circ} \\
\zeta_{2}^{\circ} \\
\zeta_{\mathbf{3}}^{\circ}
\end{array}\right]\left[\begin{array}{c}
v_{x} \\
v_{y} \\
\omega
\end{array}\right]=\left[\begin{array}{ccc}
\lambda_{1} & 0 & 0 \\
0 & \lambda_{2} & 0 \\
0 & 0 & \lambda_{3}
\end{array}\right]\left[\begin{array}{c}
\dot{\theta}_{1}^{a} \\
\dot{\theta}_{2}^{a} \\
\dot{\theta}_{3}^{a}
\end{array}\right]
$$

or in a matrix form:

$$
\mathbf{Z} \xi=\mathbf{\Lambda} \dot{\boldsymbol{\theta}} \Longrightarrow \mathbf{K} \xi=\dot{\boldsymbol{\theta}}
$$

where $\mathbf{K}=\boldsymbol{\Lambda}^{-\mathbf{1}} \mathbf{Z}$ stands for the inverse Jacobian matrix. In the above relation, $\zeta_{i}^{\circ}$ is the row vector of the three-dimensional wrench matrix, implied by the $i$ th limb. Furthermore, $\lambda_{i}$ denotes the moment of the reciprocal force around the center of the active joint in case of a revolute joint, or the projection of the force onto the direction of translation in case of a prismatic one [65]. It should be noted that $i$ is a dummy variable, which is meant to change throughout the paper.

The workspace of PMs has been investigated from different perspectives in the literature [66]. In this paper, constant-orientation workspace is considered, which consists of the set of all the possible Cartesian coordinates of a given point of the mobile platform that can be reached for a prescribed orientation [36]. The area of the workspace can be calculated numerically using discrete integration, which can be formulated as follows [64]:

$$
W=\int_{-\pi}^{\pi} A(\phi) d \phi
$$

where $A(\phi)$ is the area of the constant-orientation workspace.

From the study conducted in [55], it can be inferred that, geometrically, kinematic sensitivity is the maximum displacement or rotation of the moving platform under a unit-norm change in the joint space, which, for a PPM, can be formulated as follows:

$$
\sigma_{r_{c, f}}=\max _{\|\dot{\boldsymbol{\theta}}\|_{c}=1}\|\phi\|_{f}, \quad \sigma_{p_{c, f}}=\max _{\|\dot{\boldsymbol{\theta}}\|_{c}=1}\|\mathbf{p}\|_{\mathbf{f}}
$$

where $\sigma_{r_{c, f}}$ and $\sigma_{p_{c, f}}$ are the rotational and pointdisplacement kinematic sensitivities, respectively. Furthermore, $c$ and $f$ are the norms of the constraints functions, i.e. error in the joint space, and the norms of the pose of the moving platform, respectively. Besides, vector $\mathbf{p}$ and scalar $\phi$ stand for the variations of the position and orientation of the moving platform, respectively.

Two types of norms, namely 2- and $\infty$-norm, are frequently used in practice, since a geometric interpretation could be associated with them, which leads to four possible combinations of formulating the kinematic sensitivity, where $[35,36]$ report that $c=\infty$ and $f=2$ imply the most meaningful and reliable 
evaluation of the performance of PMs. Therefore, they are considered for the aim of this paper.

In Eq. (18), constraint $\|\dot{\boldsymbol{\theta}}\|_{c} \leq 1$ for $c=\infty$ can be rewritten as follows:

$$
\|\mathbf{K} \mathbf{x}\|_{\infty} \leq \mathbf{1}
$$

which geometrically represents a polyhedron spanned by $2^{n}$ corners $[35,36,55]$. Furthermore, $\mathbf{x}=\left[\begin{array}{ll}x & y\end{array}\right.$ $\phi]^{T}$ indicates the pose of the moving platform in the Cartesian space.

The corners of the above polyhedron can be considered as solutions to the kinametic sensitivity problem, which can be described through a system of equations, formulated as follows:

$$
\mathbf{L} \Delta \mathbf{x} \preceq \mathbf{1}_{2 n}, \quad \mathbf{L} \equiv\left[\begin{array}{ll}
\mathbf{K}^{T} & -\mathbf{K}^{T}
\end{array}\right]^{T},
$$

where sign $\preceq$ indicates that the above inequalities should be treated in a component-wise manner. The polyhedron is symmetric with respect to the origin, thus solving only $\frac{2^{n}}{2}$ inequalities suffices. PPMs only have three degrees of freedom, i.e. $n=3$, which means that the polygon has eight corners, thus one should consider four, namely, $\left(x_{i}, y_{i}\right), i=1, \ldots, 4$. Then, the kinematic sensitivity indices can be formulated as follows $[34,35]$ :

$$
\sigma_{p_{\infty, 2}}=\max _{i=1, \ldots, 4} \sqrt{x_{i}^{2}+y_{i}^{2}}, \quad \sigma_{r_{\infty, 2}}=\max _{i=1, \ldots, 4} \phi_{i}
$$

Given the fact that the above parameters always take nonnegative values, they could be confined within the interval from zero to one by taking advantage of the following substitutions:

$$
\sigma_{p_{\infty, 2}}^{\prime}=\frac{1}{1+\sigma_{p_{\infty}, 2}}, \quad \sigma_{r_{\infty, 2}}^{\prime}=\frac{1}{1+\sigma_{r_{\infty}, 2}},
$$

which are discussed in [64]. Next, averaging these values in the whole workspace of the PM gives the global kinematic sensitivity [64,67] as follows:

$$
\zeta_{\sigma_{p_{\infty}, 2}^{\prime}}=\frac{\int_{W} \sigma_{p_{\infty, 2}}^{\prime} d W}{\int_{W} d W}, \quad \zeta_{\sigma_{r_{\infty}, 2}^{\prime}}=\frac{\int_{W} \sigma_{r_{\infty},}^{\prime} d W}{\int_{W} d W}
$$

with an overall gauge of the kinetostatic performance of the PM.

The three objective functions of the MOO are expressed in Eqs. (17) and (23), which should be maximized through manipulating the geometric design parameters, schematically shown in Figure 1. The PPMs are supposed to occupy an equal area in the space. Therefore, each base triangle is assumed to be equilateral, the diameter of whose circumscribed circle is $\lambda_{a}=1$. Furthermore, the design parameters

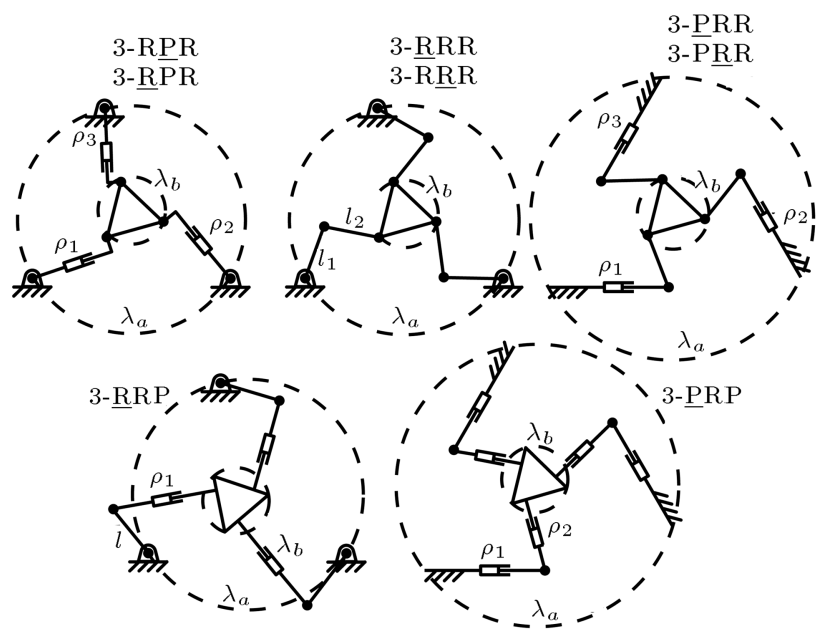

Figure 1. Eight PPMs with the corresponding geometric design parameters. The schematic is taken from [36].

should be confined within rational intervals. The legs of each PPM are supposed to be symmetric. Therefore, only the parameters associated with a single leg are considered for optimization.

As regards the constraints, the strokes of the prismatic joints are limited as follows:

$$
\begin{aligned}
& \rho_{\min }>0.1, \quad \rho_{\max }<0.75, \quad \rho_{\max }>\rho_{\min } \\
& \rho_{\max }-\rho_{\min }<\rho_{\min } \Rightarrow \rho_{\max }<2 \rho_{\min } .
\end{aligned}
$$

In order to come up with designs of practical use, the size of the moving platform, $\lambda_{b}$, should take a reasonable value with respect to the fixed size of the base. Therefore, it is constrained as follows:

$$
0.1<\lambda_{b}<\lambda_{a}=1
$$

Besides, the rigid links are confined within the following range:

$$
0.05<l<0.5 \text {. }
$$

\section{Implementation of the case study, the results and discussion}

In this section, the PPCOF is utilized by means of DE through the case-study introduced in the previous section, along with the discussion substantiating its efficiency, reliability, and robustness. For normalizing the objective functions, the outcomes of the individual SOO procedures on the same case study in [36] are used as the maximal values, where all the objective functions have practical lower bounds of zero. The best solutions obtained by the PPCOF-based MOO are presented in Table 1, where the upper bound of each objective is shown in Table 2. 
Table 1. The best solutions obtained by MOO using DE based on PPCOF.

\begin{tabular}{|c|c|c|c|c|c|}
\hline PPM & & Th & param & ters & \\
\hline 3-R $\underline{P R}$ & $\begin{array}{c}\lambda_{b} \\
0.6774\end{array}$ & $\begin{array}{c}\rho_{\min } \\
0.3818\end{array}$ & $\begin{array}{c}\rho_{\max } \\
0.7500\end{array}$ & - & - \\
\hline 3-ㅁR & $\begin{array}{c}\lambda_{b} \\
0.9611\end{array}$ & $\begin{array}{c}\rho_{\min } \\
0.3524\end{array}$ & $\begin{array}{c}\rho_{\max } \\
0.7048\end{array}$ & $\begin{array}{c}l \\
0.4888\end{array}$ & - \\
\hline 3-ㅁP & $\begin{array}{c}\lambda_{b} \\
0.8657\end{array}$ & $\begin{array}{c}\rho_{1 \mathrm{~min}} \\
0.3463\end{array}$ & $\begin{array}{c}\rho_{1 \max } \\
0.6919\end{array}$ & $\begin{array}{c}\rho_{2 \min } \\
0.3226\end{array}$ & $\begin{array}{l}\rho_{2 \max } \\
0.6370\end{array}$ \\
\hline 3 - $\underline{R} P R$ & $\begin{array}{c}\lambda_{b} \\
0.7238\end{array}$ & $\begin{array}{c}\rho_{\min } \\
0.3538\end{array}$ & $\begin{array}{c}\rho_{\max } \\
0.7066\end{array}$ & - & - \\
\hline 3-RRR & $\begin{array}{c}\lambda_{b} \\
0.8086\end{array}$ & $\begin{array}{c}l_{1} \\
0.2999\end{array}$ & $\begin{array}{c}l_{2} \\
0.4772\end{array}$ & - & - \\
\hline 3 - $\underline{R R R}$ & $\begin{array}{c}\lambda_{b} \\
0.8643\end{array}$ & $\begin{array}{c}l_{1} \\
0.3287\end{array}$ & $\begin{array}{c}l_{2} \\
0.4320\end{array}$ & - & - \\
\hline 3-P $\underline{R R}$ & $\begin{array}{c}\lambda_{b} \\
0.9572\end{array}$ & $\begin{array}{c}\rho_{\min } \\
0.3751\end{array}$ & $\begin{array}{c}\rho_{\max } \\
0.7500\end{array}$ & $\begin{array}{c}l \\
0.3230\end{array}$ & - \\
\hline 3 - $\underline{R R P}$ & $\begin{array}{c}\lambda_{b} \\
0.7466\end{array}$ & $\begin{array}{c}\rho_{\min } \\
0.1979\end{array}$ & $\begin{array}{c}\rho_{\max } \\
0.3950\end{array}$ & $\begin{array}{c}l \\
0.3427\end{array}$ & - \\
\hline
\end{tabular}

Table 2. The upper bounds of the objective functions obtained by individual SOO processes in [36].

\begin{tabular}{|c|c|c|c|}
\hline \multirow{2}{*}{ PPM } & \multicolumn{3}{|c|}{ The upper bounds } \\
\hline & $\zeta_{\sigma_{p_{\infty}, 2}^{\prime}}$ & $\zeta_{\sigma_{r_{\infty}, 2}^{\prime}}$ & $\boldsymbol{W}$ \\
\hline 3 -RPR & 0.4060 & 0.2984 & 1.2140 \\
\hline 3-PRR & 0.8856 & 0.5116 & 0.2739 \\
\hline $3-\underline{P} R P$ & 0.4797 & 0.2695 & 0.1125 \\
\hline 3 - $\underline{R} P R$ & 0.7639 & 0.7143 & 1.2199 \\
\hline $3-\overline{R R R}$ & 0.9999 & 0.9975 & 6.8974 \\
\hline 3 - $\underline{R R R}$ & 0.9927 & 0.9980 & 6.8974 \\
\hline 3 -PRR & 1.0000 & 0.9688 & 0.2739 \\
\hline 3 - $\underline{R R P}$ & 0.7260 & 0.5759 & 0.1318 \\
\hline
\end{tabular}

The optimal values of the objective functions achieved through MOO based on the PPCOF using DE are presented in Table 3, along with their normalized counterparts. The normalized values of the optimal objective functions are convincingly close to each other in case of each PPM, which shows the effectiveness of the PPCOF in accomplishing its main purpose.

From another perspective, since the PPCOF is devised such that it would properly enforce that the normalized objective functions should take similar values upon optimization, the penalty factors responsible for the latter notion in Eq. (13) should get close to zero through the optimization iterations, whose statuses at the last iteration could be checked by substituting the optimal values of the normalized objective functions into them, as shown in Table 4. The foregoing values are, indeed, approximately equal to zero, further verifying the reliability of the PPCOF in terms of preserving the desired proportions between the objective functions.

On top of that, as another major virtue of the PPCOF, no decision-making is required anymore. Nevertheless, the efficiency of the PPCOF should also be analyzed in terms of the main mission of the optimization process, namely getting the objective functions as close to their desired values as possible. In order to do so, the results can be compared with those of NSGA-II applied to the same case study in [36]. For this purpose, as the first step, the results are compared by the reasoning of the dominance described before. It has been conducted on all the eight PPMs under study, which reveals that none of the solutions returned by utilizing the PPCOF is dominated by any member of the corresponding set of Pareto-optimal solutions, meaning that the PPCOF is at least non-inferior to NSGA-II, and its result could itself be considered a member of the foregoing set.

Furthermore, the solution returned by MOO based on the PPCOF can be compared against the set of Pareto-optimal solutions through projecting the former onto the latter, which, in the context of this

Table 3. The optimal values of the objective functions, both before and after normalization, which have been found out by MOO using DE based on the PPCOF.

\begin{tabular}{|c|c|c|c|c|c|c|}
\hline \multirow{2}{*}{ PPM } & \multicolumn{3}{|c|}{ The optimal values } & \multicolumn{3}{|c|}{ The normalized values } \\
\hline & $\zeta_{\sigma_{p_{\infty}, 2}^{\prime}}$ & $\zeta_{\sigma_{r_{\infty}, 2}^{\prime}}$ & $W$ & $\zeta_{\sigma_{p_{\infty}, 2}^{\prime}}$ & $\zeta_{\sigma_{r_{\infty, 2}}^{\prime}}$ & $W$ \\
\hline 3-R $\underline{\mathrm{PR}}$ & 0.1433 & 0.0980 & 0.4234 & 0.3529 & 0.3283 & 0.3488 \\
\hline 3-PRR & 0.3503 & 0.2239 & 0.1140 & 0.3955 & 0.4376 & 0.4162 \\
\hline 3-PRP & 0.3472 & 0.1950 & 0.0814 & 0.7237 & 0.7237 & 0.7238 \\
\hline 3 - $\underline{R P R}$ & 0.2995 & 0.2832 & 0.4752 & 0.3920 & 0.3964 & 0.3896 \\
\hline $3-\mathrm{R} \underline{\mathrm{R} R}$ & 0.3536 & 0.3236 & 2.3391 & 0.3536 & 0.3244 & 0.3391 \\
\hline 3 - $\underline{R R R}$ & 0.3853 & 0.3875 & 2.6781 & 0.3882 & 0.3883 & 0.3883 \\
\hline $3-\mathrm{PRR}$ & 0.6698 & 0.5822 & 0.1732 & 0.6698 & 0.6009 & 0.6324 \\
\hline $3-\underline{R R P}$ & 0.3984 & 0.3130 & 0.0711 & 0.5487 & 0.5435 & 0.5392 \\
\hline
\end{tabular}


Table 4. The penalty factors at the last optimization iteration.

\begin{tabular}{|c|c|c|c|}
\hline \multirow{2}{*}{ PPM } & \multicolumn{3}{|c|}{ The penalty factors } \\
\hline & $\zeta_{\sigma_{p_{\infty, 2}^{\prime}}}$ & $\zeta_{\sigma_{r_{\infty, 2}^{\prime}}^{\prime}}$ & $W$ \\
\hline 3-RPR & 0.0147 & 0.0227 & 0.0084 \\
\hline $3-\underline{P R R}$ & 0.0354 & 0.0367 & 0.0000 \\
\hline $3-\underline{P R P}$ & 0.0000 & 0.0001 & 0.0001 \\
\hline $3-\underline{R P R}$ & 0.0011 & 0.0062 & 0.0051 \\
\hline $3-\mathrm{RRR}$ & 0.0222 & 0.0220 & 0.0003 \\
\hline 3 - $\underline{R R R}$ & 0.0001 & 0.0000 & 0.0000 \\
\hline $3-\mathrm{PRR}$ & 0.0995 & 0.0882 & 0.0024 \\
\hline $3-\underline{R R P}$ & 0.0108 & 0.0006 & 0.0101 \\
\hline
\end{tabular}

paper, is approximated by finding the point in the set of Pareto-optimal solutions that is closest to it. As an illustrative example, it can be imagined that the set of Pareto-optimal solutions is embraced by a hyperplane, the projection of the solution obtained by PPCOFbased MOO onto which is the point within the set that has the minimum Euclidean distance from it. Then, comparing the Euclidean distance of the two from the origin would provide a criterion for deciding which one has been more successfully attracted towards the origin for it to get minimized or pushed away from it to get maximized. The projected points are shown in Table 5 for all the PPMS, along with their normalized values. It should be noted that the foregoing points and distances between them may not make geometric sense and are just used as mathematical means for the purpose of comparison.
Now that the normalized projected point has been found, the comparison can be performed through calculating the ratio of its Euclidean norm to that of the normalized optimal point itself, which can be taken from Table 3. More clearly, this ratio is considered for evaluating the strength of the PPCOF in making the MOO maximize the objective functions, as a whole, compared to NSGA-II. The latter metric, which again may not be geometrically meaningful, is proposed for the goal of the paper and referred to as Success Rate ( $S R$ ), whose values are provided in Table 6 . It can be clearly seen from the table that $S R$ has taken values greater than 1 in nearly all the cases, averaging 1.0158. If not, $S R$ is still taking values quite close to 1 . In other words, the norm of the vector of normalized optimal values of the objective functions achieved by considering it for the MOO is higher than that of its projection onto the set of Pareto-optimal solutions obtained by NSGA-II in most cases, showing that it has been more successful than NASGA-II in pushing the objective functions' values outward from the origin, i.e. maximizing them.

One could clearly observe from the above reasoning that the proposed objective function, PPCOF, leads to an efficient, fast and handy framework for MOO scenarios with conflicting objectives. Therefore, once the objectives and parameters of the MOO are defined, the PPCOF can be readily calculated and then optimized using a typical SOO procedure, which is applicable to a variety of engineering and scientific problems similar to the case study investigated throughout

Table 5. The approximate projection of the optimal objective function points onto the corresponding sets of Pareto-optimal points obtained by NSGA-II in [36], before and after normalization.

\begin{tabular}{|c|c|c|c|c|c|c|}
\hline \multirow{2}{*}{ PPM } & \multicolumn{3}{|c|}{ The Pareto-optimal points } & \multicolumn{3}{|c|}{ The normalized values } \\
\hline & $\zeta_{\sigma_{p_{\infty}, 2}^{\prime}}$ & $\zeta_{\sigma_{r_{\infty}, 2}^{\prime}}$ & $W$ & $\zeta_{\sigma_{p_{\infty}, 2}^{\prime}}$ & $\zeta_{\sigma_{r_{\infty}, 2}^{\prime}}$ & $W$ \\
\hline 3-RPR & 0.1476 & 0.0962 & 0.4145 & 0.3636 & 0.3225 & 0.3415 \\
\hline 3-PRR & 0.3383 & 0.2303 & 0.1140 & 0.3820 & 0.4502 & 0.4162 \\
\hline 3-P्PRP & 0.3983 & 0.2021 & 0.0622 & 0.8303 & 0.7500 & 0.5527 \\
\hline 3 - $\underline{R P R}$ & 0.2984 & 0.2674 & 0.4056 & 0.3906 & 0.3743 & 0.3325 \\
\hline $3-\mathrm{R} \underline{\mathrm{RR}}$ & 0.3722 & 0.2780 & 2.1644 & 0.3723 & 0.2786 & 0.3138 \\
\hline $3-\underline{R R R}$ & 0.3258 & 0.4026 & 2.7166 & 0.3282 & 0.4034 & 0.3939 \\
\hline $3-\mathrm{PRR}$ & 0.8004 & 0.6452 & 0.1273 & 0.8004 & 0.6660 & 0.4648 \\
\hline $3-\underline{R R P}$ & 0.3989 & 0.3061 & 0.0711 & 0.5494 & 0.5316 & 0.5392 \\
\hline
\end{tabular}

Table 6. The SR values.

\begin{tabular}{ccccccccc}
\hline PPM & $3-\mathrm{RPR}$ & $3-\underline{\mathrm{PRR}}$ & $3-\underline{\mathrm{PRP}}$ & $3-\underline{\mathrm{RPR}}$ & $3-\mathrm{R} \underline{\mathrm{R} R}$ & $3-\underline{\mathrm{R} R R}$ & $3-\mathrm{PRR}$ & $3-\underline{\mathrm{R} R P}$ \\
\hline $\boldsymbol{S R}$ & 1.0018 & 0.9994 & 1.0043 & 1.0711 & 1.0475 & 1.0307 & 0.9645 & 1.0070 \\
\hline
\end{tabular}


the paper, including design and control optimization of various types of electromechanical structures, such as mannequin robots $[68,69]$, spatial mechanical manipulators [70-73] and cable-driven parallel robots [74], as well as optimization of $3 \mathrm{D}$ measurement [75], reconstruction [76], and post-processing [77] procedures utilized in the contexts of 3D simulation, modeling, and visualization.

\section{Conclusion}

This paper proposed a robust proportion-preserving composite objective function, referred to as PPCOF for multi-objective optimization, and verified its reliability and applicability through implementing it on a case study dealing with optimizing the kinematic sensitivity and workspace of planar parallel mechanisms. The PPCOF aims at alleviating the deficiencies of both preference-based and evolutionary multi-objective optimization techniques, namely requiring the preference factors as inputs from the user and performing decisionmaking, respectively. It makes the optimization algorithm look for solutions that present proportions between the resulting values of the objective functions that are roughly the same as those of the ideal ones. The objective functions, in the context of PPCOF, are normalized based on the extrema found through separate SOO processes, rather than the nominal ones, meant to exclude unrealistic marginal values. The mathematical structure of the PPCOF is such that it adds the normalized objective functions up, and then introduces penalty factors demanding that they should take values closely similar to each other, which means that all the objectives have to be given the same level of importance and prevented from ruining the results of the others. The latter would otherwise happen due to the conflicting nature of the objectives taken into account in multi-objective optimization settings. The penalty factors are treated in the same manner as the normalized objective functions themselves, implying the fact that the proportions which should be preserved bear the same level of significance as optimizing the objective functions. For constructing the penalty factors, first, the normalized objective functions are juxtaposed inside a vector, and then scaled such that the Euclidean norm of the resulting vector is equal to that of the vector of all ones with the same dimension. Then, the penalty factors are calculated by finding the absolute value of the difference between the associated values in the foregoing vector from 1 . The PPCOF could be optimized using an arbitrary single-objective optimization procedure, where differential evolution was utilized for the purpose of this paper. The results of the implementation of the case study were compared with the ones obtained by NSGA-II in a previous study. First of all, it was concluded that the solutions reported by the PPCOF are not dominated by the ones included in the set of Pareto-optimal solutions determined by NSGA-II. Besides, the strength of the PPCOF was evaluated by roughly projecting the resulting vector of the normalized optimal values of the objective functions onto the set of Pareto-optimal solutions, and comparing the Euclidean norms of them, revealing that the results were achieved by PPCOF where preferable in almost all the cases.

\section{Acknowledgment}

This research was partly funded by the Estonian Research Council Grant PUT (PUT638).

\section{References}

1. Babu, B. and Jehan, M. "Differential evolution for multi-objective optimization", The Congress on Evolutionary Computation (CEC), 4, IEEE, pp. 26962703 (2003).

2. Bandyopadhyay, S., Saha, S., Maulik, U., and Deb, K. "A simulated annealing-based multi-objective optimization algorithm: AMOSA," IEEE Transactions on Evolutionary Computation, 12(3), pp. 269-283 (2008).

3. Branke, J., Greco, J., Słowiński, R. and Zielniewicz, P. "Interactive evolutionary multi-objective optimization using robust ordinal regression", Evolutionary MultiCriterion Optimization, Springer, pp. 554-568 (2009).

4. Brockhoff, D. and Zitzler, E. "Are all objectives necessary? On dimensionality reduction in evolutionary multi-objective optimization", Parallel Problem Solving from Nature-PPSN IX, Springer, pp. 533542 (2006).

5. Brockhoff, D. and Zitzler, E. "Dimensionality reduction in multi-objective optimization: The minimum objective subset problem", Operations Research Proceedings, Springer, pp. 423-429 (2007).

6. Chattopadhyay, A. and Seeley, C.E. "A simulated annealing technique for multi-objective optimization of intelligent structures", Smart Materials and Structures, 3(2), p. 98 (1994).

7. Coello, C.C. and Pulido, G.T. "A micro-genetic algorithm for multi-objective optimization", Evolutionary Multi-Criterion Optimization, Springer, pp. 126140 (2001).

8. Collette, Y. and Siarry, P., Multi-objective Optimization: Principles and Case Studies", Springer Science \& Business Media (2013).

9. Deb, K., Thiele, L., Laumanns, M. and Zitzler, E., Scalable Test Problems for Evolutionary MultiObjective Optimization, Springer (2005).

10. Huband, S., Barone, L., While, L., and Hingston, P. "A scalable multi-objective test problem toolkit", In International Conference on Evolutionary Multi-Criterion Optimization, 3410, pp. 280-295 (2005). 
11. Deb, K. "Multi-objective optimization", Search Methodologies, Springer, pp. 403-449 (2014).

12. Deb, K. "Unveiling innovative design principles by means of multiple conflicting objectives", Engineering Optimization, 35(5), pp. 445-470 (2003).

13. Deb, K. and Datta, R. "A fast and accurate solution of constrained optimization problems using a hybrid bi-objective and penalty function approach", Congress on Evolutionary Computation (CEC), IEEE, pp. 18 (2010).

14. Golberg, D.E., Genetic Algorithms in Search, Optimization, and Machine Learning, Addison-Wesley Longman Publishing Co., Inc. (1989).

15. Deb, K. "Solving goal programming problems using multi-objective genetic algorithms", Proceedings of the Congress on Evolutionary Computation (CEC), IEEE, 1 (1999).

16. Deb, K., Multi-Objective Optimization Using Evolutionary Algorithms, John Wiley \& Sons, 16 (2001).

17. Deb, K. and Jain, S. "Running performance metrics for evolutionary multi-objective optimizations" Proceedings of the Fourth Asia-Pacific Conference on Simulated Evolution and Learning (SEAL), pp. 1320 (2002).

18. Deb, K. and Jain, S. "Multi-speed gearbox design using multi-objective evolutionary algorithms", Journal of Mechanical design, 125(3), pp. 609-619 (2003).

19. Deb, K. and Jain, H. "Handling many-objective problems using an improved NSGA-II procedure", Congress on Evolutionary Computation (CEC), IEEE, pp. 18 (2012).

20. Deb, K., Pratap, A., Agarwal, S. and Meyarivan, T. "A fast and elitist multi-objective genetic algorithm: NSGA-II", Evolutionary Computation, IEEE Transactions on, 6(2), pp. 182-197 (2002).

21. Corne, D.W., Knowles, J.D. and Oates, M.J. "The Pareto envelope-based selection algorithm for multiobjective optimization", Parallel Problem Solving from Nature PPSN VI, Springer, pp. 839-848 (2000).

22. Coello, C.A.C., Lanont, G.B., and Van Veldhuizen, D.A., Evolutionary Algorithms for Solving MultiObjective Problems, 5, New York: Springer (2007).

23. Goh, C.K. and Tan, K.C. "Evolutionary multiobjective optimization in uncertain environments", Issues and Algorithms, Studies in Computational Intelligence, 186 (2009).

24. Bagchi, T.P., Multi-Objective Scheduling by Genetic Algorithms", Springer Science \& Business Media (1999).

25. Coello, C.A.C., Aguirre, A.H. and Zitzler, E., Evolutionary Multi-Criterion Optimization (2001).

26. Marler, R.T. and Arora, J.S. "Survey of multiobjective optimization methods for engineering", Structural and Multidisciplinary Optimization, 26(6), pp. 369-395 (2004).
27. Belton, V. and Stewart, T., Multiple Criteria Decision Analysis: An Integrated Approach, Springer Science \& Business Media (2002).

28. Deb, K. and Kumar, A. "Interactive evolutionary multi-objective optimization and decision-making using reference direction method", Proceedings of the 9th Annual Conference on Genetic and Evolutionary Computation, ACM, pp. 781-788 (2007).

29. Tzeng, G.H. and Huang J.J., Multiple Attribute Decision Making: Methods and Applications, CRC Press (2011).

30. Coverstone-Carroll, V., Hartmann, J. and Mason, W. "Optimal multi-objective low-thrust spacecraft trajectories", Computer Methods in Applied Mechanics and Engineering, 186(2), pp. 387-402 (2000).

31. Deb, K., Optimization for Engineering Design: Algorithms and Examples, PHI Learning Pvt. Ltd. (2012).

32. Deb, K. and Kumar, A. "Light beam search based multi-objective optimization using evolutionary algorithms", Congress on Evolutionary Computation (CEC), IEEE, pp. 2125-2132 (2007).

33. Deb, K. and Saha, A. "Multimodal optimization using a bi-objective evolutionary algorithm", Evolutionary Computation, 20(1), pp. 27-62 (2012).

34. Daneshmand, M., Tale Masouleh, M. and Anbarjafari, G. "Kinematic sensitivity analysis of a 3-DOF decoupled translational parallel mechanism with uncertainties in the passive joints", Ubiquitous Robots and Ambient Intelligence (URAI), 12th International Conference on, IEEE, pp. 85-90 (2015).

35. Saadatzi, M.H., Tale Masouleh, M., Taghirad, H.D., Gosselin, C. and Cardou, P. "Geometric analysis of the kinematic sensitivity of planar parallel mechanisms", Transactions of the Canadian Society for Mechanical Engineering, 35, pp. 477-490 (2011).

36. Daneshmand, M., Saadatzi, M.H. and Tale Masouleh, M. "Kinematic sensitivity and workspace optimization of planar parallel mechanisms using evolutionary techniques", Robotics and Mechatronics (ICRoM), 2013 First RSI/ISM International Conference on, IEEE, pp. 384-389 (2013).

37. Storn, R. and Price, K. "Differential evolution - A simple and efficient heuristic for global optimization over continuous spaces", Journal of Global Optimization, 11, pp. 341-359 (1997).

38. Deb, K., Chaudhuri, S. and Miettinen, K. "Towards estimating nadir objective vector using evolutionary approaches", Proceedings of the 8th Annual Conference on Genetic and Evolutionary Computation, ACM, pp. 643-650 (2006).

39. Corne, D.W. and Knowles, J.D. "Techniques for highly multi-objective optimization: Some non-dominated points are better than others", Proceedings of the 9th Annual Conference on Genetic and Evolutionary Computation, ACM, pp. 773-780 (2007).

40. Srinivas, N. and Deb, K. "Multi-objective optimization using non-dominated sorting in genetic algorithms", Evolutionary Computation, 2(3), pp. 221-248 (1994). 
41. Kung, H.T., Luccio, F. and Preparata, F.P. "On finding the maxima of a set of vectors", Journal of the ACM (JACM), 22(4), pp. 469-476 (1975).

42. Jensen, M.T. "Guiding single-objective optimization using multi-objective methods", Applications of Evolutionary Computing, Springer, pp. 268-279 (2003).

43. Raquel, C.R. and Naval Jr, P.C. "An effective use of crowding distance in multi-objective particle swarm optimization", Proceedings of the rth Annual Conference on Genetic and Evolutionary Computation, ACM, pp. 257-264 (2005).

44. Vira, C. and Haimes, Y.Y., Multi-Objective Decision Making: Theory and Methodology, 8th Edn., NorthHolland (1983).

45. Miettinen, K., Nonlinear Multi-Objective Optimization, 12, Springer Science \& Business Media (2012).

46. Ehrgott, M., Multicriteria Optimization, Springer Science \& Business Media (2006).

47. Haimes, Y.Y., Lasdon, L.S. and Wismer, D.A. "On a bicriterion formulation of the problems of integrated system identification and system optimization", IEEE Transactions on Systems Man and Cybernetics, 1, pp. 296-297 (1971).

48. Daneshmand, M., Tale Masouleh, M. and Saadatzi, H. "Optimization of the kinematic sensitivity and the greatest continuous circle in the constant-orientation workspace of planar parallel mechanisms", International Journal of Robotics, Theory and Applications, 4(1), pp. 12-21 (2015).

49. Mousavi, M.A., Tale Masouleh, M. and Karimi, A. "On the maximal singularity-free ellipse of planar 3RPR parallel mechanisms via convex optimization", Robotics and Computer-Integrated Manufacturing, Elsevier, 30(2), pp. 218-227 (2014).

50. Tale Masouleh, M. "Kinematic analysis of five-DOF (3T2R) parallel mechanisms with identical limb structures", Ph.D. Thesis, Laval University (2010).

51. Saadatzi, M.H., Tale Masouleh, M., Taghirad, H.D., Gosselin, C. and Cardou, P. "Geometric analysis of the kinematic sensitivity of planar parallel mechanisms", CCToMM Conference (2011).

52. Caro, S., Bennis, F. and Wenger, P. "Tolerance synthesis of mechanisms: A robust design approach", Journal of Mechanical Design, 127, pp. 86-94 (2005).

53. Binaud, N., Caro, S. and Wenger, P. "Sensitivity comparison of planar parallel manipulators", Mechanism and Machine Theory, 45(11), pp. 1477-1490 (2010).

54. Daneshmand, M., Saadatzi, M.H., Kaloorazi, M.H.F., Tale Masouleh, M. and Anbarjafari, G. "Optimal design of a spherical parallel manipulator based on kinetostatic performance using evolutionary techniques", Journal of Mechanical Science and Technology, 30(3), pp. 1323-1331 (2016).
55. Cardou, P., Bouchard, S. and Gosselin, C. "Kinematic sensitivity indices for dimensionally nonhomogeneous Jacobian matrices", IEEE Transactions on Robotics, 26(1), pp. 166-173 (2010).

56. Merlet, J. "Jacobian, manipulability, condition number, and accuracy of parallel robots", Journal of Mechanical Design, 128, pp. 199-206 (2006).

57. Briot, S. and Bonev, I. "Are parallel robots more accurate than serial robots?", Transactions of the Canadian Society for Mechanical Engineering, 31(4), pp. $445-456$ (2007).

58. Wenger, P., Gosselin, C. and Maill, B., A Comparative Study of Serial and Parallel Mechanism Topologies for Machine Tools (1999).

59. Angeles, J. "Is there a characteristic length of a rigid-body displacement?", Mechanism and Machine Theory, 41(8), pp. 884-896 (2006).

60. Yoshikawa, T. "Manipulability of robotic mechanisms", The International Journal of Robotics Research, 4(2), pp. 3-9 (1985).

61. Stocco, L.J., Salcudean, S. and Sassani, F. "On the use of scaling matrices for task-specific robot design", IEEE Transactions on Robotics and Automation, 15(5), pp. 958-965 (1999).

62. Angeles, J., Fundamentals of Robotic Mechanical Systems: Theory, Methods, and Algorithms, Springer (2006).

63. Khan, W.A. and Angeles, J. "The kinetostatic optimization of robotic manipulators: The inverse and the direct problems", Journal of Mechanical Design, 128(1), pp. 168-178 (2006).

64. Saadatzi, M.H., Tale Masouleh, M., Taghirad, H.D., Gosselin, C. and Teshnehlab, M. "Multi-objective scale independent optimization of 3-RPR parallel mechanisms", 13th World Congress in Mechanism and Machine Science, Guanajuato, Mexico (2011).

65. Bonev, I.A., Zlatanov, D. and Gosselin, C. "Singularity analysis of 3-DOF planar parallel mechanisms via screw theory", Journal of Mechanical Design, 125, pp. 573-581 (2003).

66. Gosselin, C. "Determination of the workspace of 6DOF parallel manipulators", Journal of Mechanical Design, 112(3), pp. 331-336 (1990).

67. Saadatzi, M.H., Tale Masouleh, M., Taghirad, H.D., Gosselin, C. and Cardou, P. "On the optimum design of 3-RPR parallel mechanisms", 19th Iranian Conference on Electrical Engineering (ICEE), IEEE, pp. 16 (2011).

68. Daneshmand, M., Aabloo, A. and Anbarjafari, G. "Size-dictionary interpolation for robots adjustment", Frontiers in Bioengineering and Biotechnology, Frontiers Media SA, 3 (2015). 
69. Daneshmand, M., Aabloo, A., Ozcinar, C. and Anbarjafari, G. "Real-time, automatic shape-changing robot adjustment and gender classification", Signal, Image and Video Processing, Springer London, 10(4), pp. 753-760 (2016).

70. Tale Masouleh, M. and Gosselin, C. "Singularity analysis of 5-RPUR parallel mechanisms (3T2R)", The International Journal of Advanced Manufacturing Technology, 57(9), pp. 1107-1121 (2011).

71. Tale Masouleh, M. and Gosselin, C. "Kinematic analysis and singularity representation of 5-RPRRR parallel mechanisms", International Design Engineering Technical Conferences and Computers and Information in Engineering Conference, American Society of Mechanical Engineers, pp. 1125-1132 (2007).

72. Tale Masouleh, M., Husty, M. and Gosselin, C. "A general methodology for the forward kinematic problem of symmetrical parallel mechanisms and application to 5-PRUR parallel mechanisms (3T2R)", International Design Engineering Technical Conferences and Computers and Information in Engineering Conference, American Society of Mechanical Engineers, pp. 12791288 (2010).

73. Tale Masouleh, M. and Gosselin, C. "Singularity analysis of 5-RPRRR parallel mechanisms via Grassmann line geometry", International Design Engineering Technical Conferences and Computers and Information in Engineering Conference, American Society of Mechanical Engineers, pp. 969-978 (2009).

74. Kaloorazi, M.H.F., Esfahani, S., Tale Masouleh, M. and Daneshmand, M. "Dimensional synthesis of planar cable-driven parallel robots via interval analysis", CCToMM Symposium, IFToMM, Montreal, Quebec, Canada (2013).

75. Traumann, A., Daneshmand, M., Escalera, S. and Anbarjafari, G. "Accurate 3D measurement using optical depth information", Electronics Letters, IET, 51(18), pp. 1420-1422 (2015).

76. Valgma, L., Daneshmand, M., and Anbarjafari, G. "Iterative closest point based 3D object reconstruction using RGB-D acquisition devices", 24th Signal Processing and Communication Application Conference (SIU), IEEE, pp. 457-460 (2016).

77. Avots, E., Daneshmand, M., Traumann, A., Escalera, S. and Anbarjafari, G. "Automatic garment retexturing based on infrared information", Computers \& Graphics, Pergamon, 59, pp. 28-38 (2016).

\section{Biographies}

Morteza Daneshmand received BSc degree in Electrical Engineering, Control, from the Tehran Polytechnic (Amirkabir) University of Technology, Tehran, Iran. His thesis was entitled "Implementation of Distributed Formation Control of Multi-agent Systems on e-puck Mobile Robots". Afterward, he received his MSc in Mechatronics Engineering from the University of Tehran, Tehran, Iran. His thesis was entitled "Kinematic Sensitivity and Workspace Optimization of Planar Parallel Mechanisms". Currently, since September 2014, he is a PhD student at the University of Tartu, Tartu, Estonia, working on "3D Modeling and Visualization for a Realistic, Virtual Fitting Room". He has been involved in numerous national and international projects. He has been the Chair of the University of Tartu IEEE Student Branch since its establishment in December 2015.

Mehdi Tale Masouleh received B Eng, MSc and $\mathrm{PhD}$ degrees in Mechanical engineering (Robotic) from Laval University, Qubec, Canada, in 2006, 2007, and 2010, respectively. He is currently a Faculty Member of School of Electrical and Computer Engineering, University of Tehran. He is also the director of HumanRobot Interaction Laboratory, known as TaarLab for its Persian abbreviation. His research interests are kinematics, dynamic and design of serial and parallel robotic systems, humanoid, mobile robots and optimization techniques (i.e. interval analysis and convex optimization) for robotic applications. $\mathrm{He}$ is also director of a national-level project for a haptic dental simulator.

Mohammad-Hossein Saadatzi is a PhD student at Mechanical Engineering Department of Colorado School of Mines. He did his undergraduate in Robotics Engineering and his masters in Mechatronics Engineering, both in Iran. His academic background and interests are in the interdisciplinary fields of robotics, control and biomechanics. For his bachelors and masters final projects, he has worked on the kinematics, dynamics, design and control of serial and parallel mechanisms, results of which have been published in several prestigious journals and conference papers. He also received the IEEE Iran sections Best MSc Thesis award of the year 2012 .

Cagri Ozcinar received MSc (Hons.) and PhD degrees in Electronic Engineering from the University of Surrey, UK, in 2010 and 2015, respectively. Afterwards, for one year then he has been with the Multimedia Group of Télécom ParisTech, Paris, France. He is currently a research follow at School of Computer Science and Statistics, Trinity College Dublin. His research interests include HDR/WCG and 3D/FTV/MV video processing, compression, and delivery techniques.

Gholamreza Anbarjafari is heading the intelligent Computer Vision (iCV) research group in the Institute of Technology at the University of Tartu. He is also the supervisor of Philosopher, the Estonian Robocup team of University of Tartu. He is an IEEE Senior member and the Vice Chair of Signal Processing/Circuits and 
Systems/Solid-State Circuits Joint Societies Chapter of IEEE Estonian section. He has got Estonian Research Council Grant (PUT638) in January 2015 and has been involved in many international industrial projects such as AIDesign, A.G.E., iRental and Virtual Fitting Room by Fits.Me Rakutan. He is expert in computer vision, human-robot interaction, graphical models and artificial intelligence. His work in image super resolution has been selected for the best paper award in 2012 by Electronics and Telecommunications
Research Institute (ETRI) Journal, South Korea. He has supervised $7 \mathrm{MSc}$ students and $5 \mathrm{PhD}$ students. He has published over 90 scientific works. He has been in the organizing committee and technical committee of IEEE Signal Processing and Communications Applications Conference in 2013, 2014 and 2016 and TCP of conferences such as ICOSST, ICGIP, and SIU. He is organizing a challenge and a workshop on Dominant and Complementary Emotion Recognition Using Micro Emotion Features and Head-Pose Estimation in FG17. 